\title{
AUTONOMÍA Y HETERONOMÍA DE LA EDUCACIÓN MILITAR. UN ANÁLISIS DE LOS PROCESOS DE EVALUACIÓN INSTITUCIONAL EN LOS INSTITUTOS UNIVERSITARIOS DE LAS FUERZAS ARMADAS ARGENTINAS
}

\author{
Germán Soprano *
}

Recebido: 31 jul. 2012

Aprovado: 29 out. 2012

*Universidad Nacional de Quilmes y de la Universidad Nacional de La Plata. La Plata, Argentina. Contato: gsoprano@unq.edu.ar

Resumen: A comienzos de los años 1990, las Fuerzas Armadas Argentinas tenían institutos universitarios que, además de ofrecer la tradicional formación básica y perfeccionamiento para sus oficiales, promovieron estudios para civiles y militares. A partir de una revisión de las principales recomendaciones de los pares evaluadores de la CONEAU en los procesos de evaluación institucional de los Institutos Universitarios de las Fuerzas Armadas, analizaremos las continuidades y cambios en el sistema de educación militar y sus grados de autonomía y heteronomía como resultado de su participación en esos procesos.

Palabras clave: Educación militar. Evaluación institucional. Autonomía. Heteronomía.

\author{
AUTONOMY AND HETERONOMY OF MILITARY EDUCATION. \\ AN ANALYSIS OF THE INSTITUTIONAL EVALUATION PROCESSES IN \\ THE UNIVERSITIES INSTITUTES OF THE ARGENTINE ARMED FORCES
}

Abstract: At the beginning of the 1990s, the Argentine Armed Forces had university institutes that, in addition to the traditional basic training and further education for its officers, promoted studies for civil and military personnel. Based on a review of the main recommendations of CONEAU's evaluators in processes of institutional evaluation in University Institutes of the Armed Forces, we will analyze the continuities and changes in the military education system and their degrees of autonomy and heteronomy as a result of their participation in the process.

Key words: Military education. Institutional evaluation. Autonomy. Heteronomy.

\author{
AUTONOMIA E HETERONOMIA DA EDUCAÇÃO MILITAR. \\ UMA ANÁLISE DOS PROCESSOS DE AVALIAÇÃO INSTITUCIONAL NOS \\ INSTITUTOS UNIVERSITÁRIOS DAS FORÇAS ARMADAS ARGENTINAS
}

Resumo: No início da década de 1990, as Forças Armadas argentinas tiveram institutos universitários que, além de oferecer a formação básica tradicional e o aperfeiçoamento para seus oficiais, promoveram estudos para civis e militares. A partir de uma revisão das principais recomendações dos pares avaliadores da CONEAU em processos de avaliação institucional dos Institutos Universitários das Forças Armadas, analisaremos as continuidades e mudanças no sistema de educação militar e do seu grau de autonomia e heteronomia como resultado de sua participação nestes processos.

Palavras chave: Educação militar. Avaliação institucional. Autonomía. Heteronomía. 


\section{INTRODUCCIÓN}

Como señalaron Guillermo O'Donnell, Philippe Schmitter y Laurence Whitehead (1994), la crisis por colapso del régimen militar argentino eliminó -fundamentalmente con el sometimiento de la última rebelión militar "carapintada" en diciembre de 1991- cualquier incidencia relevante de las Fuerzas Armadas en la orientación de la política nacional y, en consecuencia, restringió su capacidad para instalar unilateralmente intereses sectoriales en la política de defensa. A esto debemos sumar la voluntaria aceptación del principio de conducción civil de la defensa y de su instrumento militar asumida por oficiales y suboficiales en actividad durante los últimos veinte años. Ahora bien, no obstante este cuadro de situación, hasta la gestión de Nilda Garré (2005-2010) en el Ministerio de Defensa, las Fuerzas Armadas Argentinas mantuvieron considerables grados de autonomía en la conducción de la defensa y, en particular, en las decisiones relativas al diseño de políticas, gestión y evaluación de la educación militar. En este sentido, como señalan Ernesto López (2004), Pion-Berlin (2008) y Marcelo Saín (2010), en el escenario latinoamericano el caso argentino es expresivo de una situación en la cual las Fuerzas Armadas están subordinadas al poder civil, pero donde este último no ha ejercido el control civil o, más ampliamente, el gobierno político de la defensa ${ }^{1}$. Sólo desde fines de 2005 puede considerarse que el Ministerio de Defensa han diseñado y puesto en práctica decisiones que promovieron normativas y procesos que dieron lugar a una efectiva conducción civil en materia de estrategia, doctrina, planeamiento, organización y funciones de las Fuerzas Armadas (MONTENEGRO, 2007; SAÍN, 2010).

En relación con el sistema de educación militar y, en particular, con la formación básica y capacitación de los oficiales, a comienzos de la década de 1990, la conducción de las Fuerzas Armadas crearon los Institutos Universitarios de las Fuerzas Armadas (IUFFAA) integrados por unidades académicas destinadas no sólo a ofrecer la educación que históricamente brindaron a oficiales en academias militares y en Escuelas Superiores de Guerra, sino también para promover carreras de grado (Tecnicaturas, Licenciaturas e Ingenierías) y carreras de posgrado (Especializaciones y Maestrías) cursadas por militares y civiles. La sanción de la Ley de Educación Superior (LES) No 24.251 (1995)

1 Paula Canelo (2011) sostiene, a diferencia de esta interpretación dominante en los estudios sobre relaciones civiles-militares en la Argentina, que la subordinación militar en la década de 1990 a las autoridades civiles democráticas fue condicionada; al tiempo que considera que en la actualidad existen indicadores que muestran que las Fuerzas Armadas no están todavía suficientemente integradas al orden democrático. 
en Argentina favoreció la consolidación de esas transformaciones. Pero no debe desconsiderarse el hecho de que esos cambios se desplegaron, por un lado, en un contexto nacional determinado por la búsqueda de nuevas formas de reconocimiento social para las Fuerzas Armadas y por la vigencia de la Ley de Defensa Nacional No 23.554 (1988) y la Ley de Reestructuración de las Fuerzas Armadas No24.948 (1998); y, por otro lado, en un escenario internacional signado por la tendencia a otorgar títulos universitarios como parte de la formación básica de los oficiales² .

Teniendo en cuenta ese marco político, normativo y educativo nacional e internacional, el proceso de cambio en el sistema educativo castrense fue orientado casi exclusivamente por decisiones de la conducción de las Fuerzas Armadas, pues la conducción civil de la defensa sólo se involucró más activamente con la creación de la Subsecretaría de Formación del Ministerio de Defensa en $2007^{3}$. Hasta entonces, la autonomía de las Fuerzas sobre la educación militar se confrontaba, por un lado, con la intervención heterónoma establecida por normativas, autoridades y funcionarios del Ministerio de Educación en relación con el reconocimiento y validez nacional de los títulos otorgados por los IUFFAA. Y, por otro lado, por las autoridades, técnicos y pares evaluadores de la Comisión Nacional de Evaluación y Acreditación Universitaria (CONEAU) que intervienen en la evaluación institucional y la acreditación de carreras.

A tal efecto, presentaremos, en primer término, los fines educativos y el modo en que los IUFFAA se inscribieron en el contexto de transformaciones de la educación superior argentina desde 1990. En segundo lugar, abordaremos la organización de las unidades académicas que integran los IUFFAA y sus actuales ofertas de carreras de grado y posgrado. En tercer lugar, señalaremos las principales observaciones expuestas por las comisiones de pares evaluadores de la CONEAU en el marco de los procesos de evaluación institucional externa y acreditación de carreras de los IUFFAA. El trabajo espera ser

2 La educación militar en la Unión Europea no se ha sustraído a la internacionalización y regionalización de saberes y prácticas universitarias debiendo, por ende, adecuar la oferta educativa de sus tradicionales academias a los acuerdos de Bologna. Así pues, en Francia los cadetes obtienen el grado militar inicial como teniente (o equivalente) y un Master; en Alemania un Bachillerato Universitario o Master en Artes, Ciencias o Ingeniería; y en España un título de grado en Ingeniería, Ciencias Económicas, Ciencias Empresariales o en Relaciones Internacionales. Por su parte, en los Estados Unidos los cadetes egresan con un título de Bachelor of Science.

3 La Subsecretaría de Formación depende de la Secretaría de Estrategia y Asuntos Militares que coordina y articula las políticas de formación, capacitación y perfeccionamiento del personal de las Fuerzas Armadas. En el cumplimiento de sus funciones esta Subsecretaría tiene por interlocutores naturales a los Directores Generales de Educación del Ejército, Armada y Fuerza Aérea, de los cuales dependen el Instituto de Educación Superior del Ejército (IESE), el Instituto Universitario Naval (INUN) y el Instituto Universitario Aeronáutico (IUA). 
un aporte empírico original al conocimiento de un tema todavía no explorado por los estudios en educación superior en la Argentina, cual es el análisis de la incorporación de la formación y capacitación militar al sistema universitario $y$, en especial, la indagación sobre los procesos de evaluación institucional de los IUFFAA ${ }^{4}$.

\section{UNIDADES ACADÉMICAS Y OFERTAS EDUCATIVAS DE LOS IUFFAA}

Las Fuerzas Armadas Argentinas cuentan con tres institutos universitarios: El Instituto de Enseñanza Superior del Ejército (IESE), el Instituto Universitario Naval (INUN) y el Instituto Universitario Aeronáutico (IUA). Hasta la sanción de la LES los IUFFAA se regían por la Ley de Universidades Provinciales $\mathrm{N}^{\circ} 17.778$ (1968) y la Resolución $\mathrm{N}^{\circ} 2.024$ (1990) del Ministerio de Educación. La Ley de Educación Superior los define en su artículo 27 como institutos universitarios, pues, a diferencia de las universidades, desarrollan sus actividades en una sola área o campo disciplinario ${ }^{5}$.

La Ley de Reestructuración de las Fuerzas Armadas planteó restricciones a la oferta educativa castrense estableciendo que: 1) Será provista a través del Ministerio de Defensa, el Estado Mayor Conjunto, Ejército, Armada y Fuerza Aérea, con coordinación y supervisión del Ministerio. 2) Su finalidad será brindar educación profesional, según las necesidades operacionales específicas del cargo, rol y función militar a desempeñar. 3) Se le designarán fondos para la formación y capacitación militar específica y en defensa, evitando la superposición de esfuerzos y de recursos humanos. 4) Estará en consonancia con el sistema educativo nacional, evitando el costo de formar y capacitar profesionales que puedan ser reclutados del mismo o proporcionar títulos de grado que puedan cursarse en otras instituciones de educación superior del ámbito civil ${ }^{\text {. }}$.

4 Este trabajo es un resultado parcial de las tareas realizadas como investigador del CONICET y como director de un proyecto radicado en la Universidad Nacional de Quilmes que tiene por objeto el análisis comparado de los procesos de formación y configuración profesional en civiles y militares.

5 Carlos Pérez Rasetti (2008) considera que la denominación como instituto universitario no es acertada para los institutos militares, en la medida en que la formación militar involucra múltiples áreas o disciplinas científicas. Su especificidad está definida por su función como instrumento de la defensa y por su condición heterónoma como monopolio del Estado nacional.

6 Las academias militares de formación básica de oficiales ofrecen títulos a sus egresados con validez nacional, los cuales permiten a sus egresados acceder a estudios de postgrado en universidad nacionales y privadas y, eventualmente, al retirarse de la carrera militar incorporarse al mercado de trabajo civil con un título de grado universitario. El CMN otorgó a los cadetes del cuerpo comando títulos de Bachiller Universitario en Relaciones Internacionales y Bachiller Universitario en Ingeniería (entre 1992 y 1996), Licenciado en Administración (1997/2005), Licenciado en Matemática Aplicada 
El Instituto de Enseñanza Superior del Ejército (IESE) fue creado en 1990. Del mismo dependen las siguientes unidades académicas de nivel superior: el Colegio Militar de la Nación (creado en 1869), la Escuela Superior de Guerra (1900) y la Escuela Superior Técnica (1930). En el Colegio Militar de la Nación se realiza la formación básica de los futuros oficiales y actualmente egresan con el grado de subteniente y el título de Licenciado en Conducción y Gestión Operativa (desde 2005). La Escuela Superior de Guerra del Ejército, además, de capacitar a los oficiales de Estado Mayor, ofrece posgrados para civiles y militares: Maestría en Estrategia y Geopolítica, Maestría en Historia de la Guerra y Especialización en Derecho Militar. La Escuela Superior Técnica tradicionalmente formó a los ingenieros militares, pero desde 1992 también a civiles en la Tecnicatura en Geomática, las Ingenierías Electrónica, Civil, Química, Mecánica (armamentos, mecánica y automotores), las Especializaciones en Criptografía y Seguridad, Gestión Ambiental, Teleinformática, Sistemas de Control, Desarme y no proliferación de armas de destrucción masiva, Mantenimiento, Materiales Explosivos, y la Maestría en Seguridad e Higiene Ocupacional.

El Instituto Universitario Naval (INUN) fue creado en 1991. Cuenta con las siguientes unidades académicas de nivel superior: la Escuela Naval Militar (1872), la Escuela Superior de Guerra Naval (1934), la Escuela Superior de Oficiales de la Armada (1904), la Escuela Superior de Técnicas y Tácticas, la Escuela Nacional de Náutica y la Escuela de Ciencias del Mar. En la Escuela Naval Militar se realiza la formación básica de los futuros oficiales, egresando con el grado de guardiamarina y el título de Licenciado en Recursos Navales para la Defensa (desde 2007), Licenciado en Sistemas Aéreos y Aeroespaciales (desde 2008). La Escuela Superior de Guerra Naval, además de capacitar a los oficiales de estado mayor, ofrece las Maestrías en Estudios Estratégicos, en Gestión Logística y en Dirección de las Organizaciones, así como un posgrado en Intereses Marítimos que también pueden ser cursados por civiles. La Escuela de Oficiales de la Armada ofrece estudios de grado para los oficiales de la Armada las Licenciaturas en Sistemas Navales, en Sistemas Navales

(1997/2000), Licenciado en Conducción y Gestión Operativa (desde 2005). Por su parte, la Escuela Naval Militar títulos de Bachiller Universitario en Sistemas Navales, Licenciado en Administración de los Recursos Navales para la Defensa (1996/2000) y Licenciado en Recursos Navales para la Defensa (desde 2007). Por último, la Escuela de Aviación Militar títulos de Bachiller Universitario en Sistemas Aéreos y Aeroespaciales (1996/2007) y Licenciado en Sistemas Aéreos y Aeroespaciales (desde 2008). En diciembre de 2011 por resolución del Ministerio de Defensa se modificaron los planes de estudios y el régimen interno de la formación básica de los oficiales del Ejército, Armada y Fuerza Aérea. Esa reforma está en el presente en proceso de implementación por las actuales autoridades del Ministerio de Defensa y las Direcciones Generales de Educación de las Fuerzas Armadas. 
Infantería de Marina y en Sistemas Navales Aéreos y los cursos de artillería, armas submarinas, comunicaciones, máquinas y electricidad, infantería de marina, cursos aplicativos para oficiales navales y de infantería de marina y cursos de estado mayor para oficiales del cuerpo profesional. Asimismo, posee cursos de posgrado para civiles y militares en Dirección en organizaciones, Logística, Análisis operativo, Análisis de sistemas automatizados para el desarrollo de las operaciones militares, Análisis de sistemas automatizados de gestión para la defensa y en mantenimiento (mecánico, eléctrico, motores diesel navales e industriales y sistemas eléctricos). Finalmente, en la Escuela Nacional de Náutica los civiles cursan las Licenciaturas en Transporte Marítimo y en Plantas Propulsoras y, en la Escuela de Ciencias del Mar, la Licenciatura en Cartografía y cursos de Meteorología, Hidrografía y Oceanografía.

El Instituto Universitario Aeronáutico (IUA) fue creado en 1971 y tuvo su antecedente en la Escuela de Ingeniería Aeronáutica fundada en 1947. Su denominación actual data de 1993. Las unidades académicas de nivel superior asociadas al IUA son: la Escuela de Aviación Militar (1912), la Escuela Superior de Guerra Aérea (1944), el Instituto Nacional de Derecho Aeronáutico y Espacial (1947) y el Instituto Nacional de Medicina Aeronáutica y Espacial (1945). En la Escuela de Aviación Militar se desarrolla la formación básica de los oficiales, egresando como alférez y el título de Licenciado en Sistemas Aéreos y Aeroespaciales (desde 2008). La Escuela Superior de Guerra Aérea capacita a los oficiales de estado mayor y cursos para civiles en poder aeroespacial. El IUA ofrece en sus Facultades de Ingeniería y en la de Ciencias de la Administración carreras de grado (en modalidad presencial, semi-presencial y a distancia) para civiles y militares (oficiales y suboficiales) como las Ingenierías en Sistemas, en Informática; en Electrónica, en Mecánica Aeronáutica y en Telecomunicaciones; las Licenciaturas en Administración, en Logística y en Recursos Humanos; la carrera de Contador Público; y los posgrados como la Maestría en Ciencias de la Ingeniería y las Especializaciones en Análisis de la Inteligencia Estratégica, Sistemas Embebidos y en Seguridad Informática. Por su parte, el Instituto Nacional de Derecho Aeronáutico y Espacial ofrece cursos de posgrado en Derecho Aeronáutico y Espacial para abogados y un diploma en Género y Gestión Institucional para civiles y militares. Y en el Instituto Nacional de Medicina Aeronáutica y Espacial cursos en medicina aeronáutica y espacial para civiles y militares.

Una revisión de las carreras de grado y posgrado brindadas por los IUFFAA permite constatar que no siempre se cumplimenta taxativamente la normativa fijada por la Ley de Reestructuración de las Fuerzas Armadas, fun- 
damentalmente, en lo atinente a su prescripción de evitar que el sistema educativo castrense se superponga o compita con ofertas educativas del sistema universitario nacional, tal como puede constatarse en relación con la carrera de Contador Público y las Licenciaturas en Administración, en Logística y en Recursos Humanos de la Facultad de Ciencias de la Administración del IUA. En otros casos, como las ofertas de grado y posgrado en ingenierías, sistemas, telecomunicaciones, cartografía, hidrografía, oceanografía, estrategia y geopolítica, historia de la guerra, derecho militar, medicina y derecho aeronáutico o espacial, $u$ otras, se trata de campos del conocimiento en los cuales las Fuerzas Armadas tienen amplia trayectoria profesional y en investigación, por ende, aunque puedan implicar una duplicación de la oferta educativa que el Estado nacional ofrece a la sociedad, no constituirían necesariamente un desvío respecto de la norma vigente (si bien esto suele ser objeto de polémica) en la medida en que son consideradas por los IUFFAA como carreras que contribuyen a la formación de recursos humanos y de conocimientos teóricos y prácticos para la defensa nacional. Aún así, algunos analistas del sistema de educación superior como Carlos Pérez Rassetti (2008) caracterizan estas últimas ofertas de los IUFFAA como innecesariamente superpuestas con la de las universidades nacionales. Por último, cabe destacar que las carreras de grado ofertadas por los IUFFAA no son gratuitas (a diferencia de lo que ocurre legalmente con las ofertas de grado de las universidades nacionales), de modo que los estudiantes civiles que las cursan y los militares que las realizan por fuera de su plan de carrera profesional en las Fuerzas deben abonar matrícula y cuotas para poder acceder y desarrollar sus estudios en ellos. No ocurre lo mismo, por cierto, con las carreras de grado asociadas a la formación básica del oficial (las Licenciaturas del CMN, la ESNM y la EAM) ni con las capacitaciones ulteriores brindas en las escuelas técnicas y en las escuelas de guerra y que son parte de su plan de carrera.

\section{LA LEY DE EDUCACIÓN SUPERIOR, LA CONEAU Y LOS IUFFAA}

Los fundamentos que orientaron el diseño e implementación de la LES en 1995 tuvieron por referencia políticas internacionales de mejora de la calidad de la enseñanza promovidas por organismos multilaterales de crédito: estímulo a la diferenciación y competencia inter-institucional y al interior del sistema, crecimiento de la oferta privada, obtención de recursos no provistos por el presupuesto estatal, políticas de equidad y redefinición de las formas 
de gobierno y gestión 7 . Y si bien es dado mencionar la incidencia de estas orientaciones de corte neoliberal, también se impuso el peso de las tradiciones ideológicas, políticas y académicas universitarias argentinas de larga duración. Los cambios más notorios se registraron en la incorporación de la educación superior no universitaria al cuerpo de la Ley, la creación de instancias de coordinación interuniversitaria, la desregulación e incentivos salariales a los docentes, así como en el planteo de dos orientaciones que pretendían reforzar la autonomía institucional: por un lado, la habilitación para el cobro de aranceles $\mathrm{y}$, por otro, la afirmación de un criterio de responsabilidad social en el ejercicio de la autonomía asociadas a la creación de la CONEAU, los procesos de evaluación institucional, la acreditación de carreras, el reconocimiento de títulos y la habilitación de las profesiones reguladas por el Estado pues comprometen de modo directo "la salud, la seguridad, los derechos, los bienes o la formación de los habitantes".

De este modo, la evaluación y la acreditación se erigieron como núcleos clave en la definición de las políticas universitarias de un nuevo "Estado evaluador" (NEAVE, 2001; ALTBACH; MCGILL PETERSEN, 2000; KROTSCH; PUIGGRÓS, 1994). Como esas nuevas orientaciones de las políticas universitarias no operaron ex nihilo, en el caso argentino las transformaciones de la educación superior estarían más bien asociadas a un proceso de reconfiguración del rol interventor del Estado nacional, antes que operando como un mero y unilateral proceso de privatización, mercantilización, segmentación y desregulación del sistema (KROTSCH; $2001)^{8}$. La incorporación de las políticas de evaluación por parte de los actores

7 Adriana Chiroleu (2007) sostiene que las políticas de educación superior en Argentina se caracterizaron por: 1) Diversificar y segmentar la oferta y demanda educativa y aumentar la escolarización en el sistema en respuesta a nuevas demandas de calificación laboral y por presiones en el acceso de diferentes sectores sociales. 2) Disponer de fondos públicos insuficientes para una demanda en expansión que, por decisiones políticas o consecuencia de restricciones presupuestarias, fue crecientemente cubierta por ofertas privadas. 3) Favorecer una internacionalización motorizada por estímulos mercantiles y en beneficio de los países capitalistas centrales. 4) Introducir lógicas y prácticas de competencia mercantil en las universidades públicas y orientar la formación profesional, investigación y transferencia en función de demandas del mercado. 5) Promover políticas de accountability que plantearon conflictos a la autonomía universitaria y la libertad de cátedra. 6) Reducir la proporción de docentes con dedicación completa y aumentar los contratados a término, fragmentándolos y diferenciándolos como cuerpo académico. Para Antonio Camou (2002) esas reformas en la educación superior argentina no dieron lugar a una unilateral tendencia a la privatización y mercantilización (como sí ocurrió con las empresas estatales), pues también se produjo una notable expansión de la oferta educativa pública y una re-regulación estatal del sistema.

8 De acuerdo con Pedro Krotsch: "La nueva racionalidad se orienta a promover la diferenciación horizontal y vertical y la creciente sensibilización de las instituciones al mercado de distinciones y prestigio [...] Es en este contexto que adquiere importancia estratégica la evaluación, el mercado y los mecanismos de regulación en el nuevo diseño institucional previsto por la Ley de Educación Superior. Los organismos y mecanismos de regulación se convierten así en elemento indispensable de un sistema 
universitarios siguió un curso en absoluto lineal, pasando por diversas instancias de rechazo abierto, resistencia, negociación, apropiación y resignificación conforme al modo en que fueron procesadas en distintas universidades, en sus unidades académicas (Facultades, Departamentos, carreras), por grupos disciplinares, corporativos y/o políticos (KROTSCH; ATAIRO; VARELA, 2007).

La CONEAU fue creada en 1995 en el marco de un Programa de Reforma de la Educación Superior, financiado con fondos del Banco Mundial y ejecutado con participación de la Secretaría de Políticas Universitarias (CAMOU, 2007; PEÓN; PUGLIESE, 2004). El artículo 44 de la LES establece los criterios para la definición e implementación de procesos de autoevaluación y de evaluación externa de las universidades e institutos universitarios. La autoevaluación estaría destinada a "asegurar el funcionamiento de instancias internas de evaluación institucional, que tendrán como objeto analizar los logros y las dificultades en el cumplimiento de sus funciones, así como sugerir medidas para su mejoramiento". La evaluación externa es complementaria y posterior a la autoevaluación; se produce tomando como referencia los "objetivos definidos por cada institución" y abarca las "funciones de docencia, investigación y extensión, y en el caso de las instituciones universitarias nacionales, también la gestión institucional”. Carlos Mazzola (2008) señala que el recurso a la autoevaluación prescribe y pone en práctica el principio de autonomía de las instituciones universitarias; en tanto que el de la evaluación externa da cuenta de la noción de accountability o del principio de legítimo derecho que posee el Estado en un régimen republicano y democrático de velar por el cumplimento de los objetivos que la sociedad asigna al sistema de educación superior y, en particular, a las universidades públicas.

Desde comienzos de la década de 1990, las experiencias destinadas a promover iniciativas por inscribir o incorporar lógicas y prácticas universitarias en los procesos de enseñanza no sólo han sido a propuesta de la conducción de las Fuerzas Armadas y el Ministerio de Defensa, sino una orientación resultante de la participación (necesaria) de los IUFFAA en los procesos de evaluación y acreditación (institucionales y de las carreras ofertadas) de la CONEAU. En este sentido, la adecuación de la oferta de las carreras y la organización institucional castrense a los estándares de calidad educativa nacionales, también operaron como determinaciones que modificaron sus

que se pretende sea el más numeroso, diverso y competitivo en términos sectoriales e institucionales: el orden de las normas, la evaluación y el control deberían permitir así estabilizar las tendencias que por otro lado presionan hacia la fragmentación, el desorden y la anomia del mercado" (2001, p. 172). 
proyectos y actividades. Sin embargo, estas determinaciones bien pudieron ser compensadas y/o contrarrestadas por la agencia de concepciones y prácticas educativas tradicionales fuertemente establecidas 9.

Esos procesos de evaluación institucional y de acreditación de sus carreras -concretados desde la segunda mitad de la década de 1990- obraron como determinaciones que heteronomizaron el control militar sobre la educación castrense. Más recientemente, esa tendencia quedó subrayada por las orientaciones impartidas por el gobierno civil de la defensa en relación con los proyectos institucionales y curriculares del IESE, INUN y IUA y, en particular, de sus academias de formación básica de oficiales, pues a instancias de la intervención de la Subsecretaría de Formación se abrió un proceso de reformulación de sus planes de estudio y de la sociabilidad del régimen de internado de esas tradicionales academias militares, al tiempo que se dio impulso a la elaboración de nuevos estatutos universitarios y de un estatuto del personal docente civil de los IUFFAA ${ }^{10}$. Así pues, si hasta fines del siglo XX el Ministerio de Educación de la Nación gravitaba como una determinación heterónoma sobre esos institutos fijando pautas generales de diseño curricular y carga horaria de los planes de estudio de las Licenciaturas para garantizar la validez nacional de sus títulos; desde 1997, además, la CONEAU y las recomendaciones de sus comisiones de pares evaluadores cobraron relevancia en las decisiones adoptadas por las autoridades educativas castrenses y, últimamente, por las civiles del Ministerio de Defensa.

La LES establece en su artículo 40 que las instituciones universitarias tienen autonomía para definir qué carreras de grado y posgrado ofertarán y poseen el monopolio para otorgar títulos de grado de licenciado y otros títulos profesionales equivalentes, así como títulos de posgrado. Los IUFFAA poseen tal potestad, pero su efectivo ejercicio es más limitado que en otras universidades públicas, toda vez que requiere de la anuencia de las Direcciones de Educación de su respectiva Fuerza y la del Ministerio de Defensa. Una vez cumplimentadas estas instancias de decisión, tal como determina el artículo 41 de la LES, el reconocimiento oficial de los títulos expedidos por los IUFFAA debe

9 En esta última línea de interpretación -que destaca la autonomía castrense en el gobierno de su sistema educativo- avanza el análisis de Máximo Badaró (2009) identifica dificultades en la concepción de la formación de los cadetes del CMN, en la organización institucional y en la sociabilidad del régimen de internado que funda el modelo de academia, que constituyen obstáculos relevantes para la incorporación de lógicas y prácticas universitarias. Un análisis comparado de la formación básica de los oficiales y, en particular, de su dimensión moral, han sido efectuados en Frederic, Soprano et al (2010) y Frederic y Soprano (2011).

10 Este último estatuto también comprende a los militares en situación de retiro que dictan clases en los IUFFAA. 
cumplimentar los requisitos demandados por el Ministerio de Educación de la Nación a fin de garantizar que los mismos serán oficialmente reconocidos y tendrán validez nacional ${ }^{11}$. Los títulos de grado de las carreras ofertadas por los IUFFAA, como los de cualquier institución universitaria, certifican la formación académica recibida y habilitan al ejercicio profesional respectivo. En el caso de las carreras que asociadas a la formación básica de los futuros oficiales de las Fuerzas Armadas, sin embargo, su ejercicio profesional está restringido, como ocurre en otros países, al ámbito del instrumento militar del Estado nacional; en tanto que los militares (oficiales o suboficiales) que cursan carreras de grado que también son ofertadas para civiles en los IUFFAA, sólo podrán ejercer en sus esos campos profesionales en el mercado laboral civil una vez que pasen a retiro, se den o sean dados de baja de la Fuerza. Estas restricciones constituyen un buen ejemplo del modo en que la autonomía académica e institucional de los institutos universitarios castrenses está limitada por su inscripción en la organización de cada Fuerza y por su relación con el Ministerio de Defensa, siendo, en este sentido, un caso excepcional como el de los institutos universitarios de las fuerzas de seguridad federales ${ }^{12}$.

También en lo que respecta al capítulo sobre instituciones universitarias nacionales y, en especial, en sus artículos sobre los órganos de gobierno, los IUFFAA presentan especificidades que los diferencian de las instituciones correspondientes a esta categoría. El artículo 77 de la LES, establece que las autoridades de los institutos universitarios son elegidas de acuerdo a los criterios que sus estatutos especifiquen ${ }^{13}$. Por tanto, en el caso de los IUFFAA

11 De acuerdo con el artículo 42 de la LES: "Los respectivos conocimientos y capacidades que tales títulos certifican, así como las actividades para las que tienen competencia sus poseedores, serán fijados y dados a conocer por las instituciones universitarias, debiendo los respectivos planes de estudio respetar la carga horaria mínima que para ello fije el Ministerio de Cultura y Educación, en acuerdo con el Consejo de Universidades".

12 Los IUFFAA -a diferencia de otras instituciones universitarias- carecen de autonomía para: a) dictar y reformar sus estatutos; b) definir sus órganos de gobierno, funciones, integración y autoridades; c) administrar bienes y recursos; d) crear carreras de grado y posgrado; e) formular y desarrollar planes de estudio, investigación y extensión; f) otorgar grados académicos y títulos habilitantes; g) impartir enseñanza con fines de experimentación, innovación pedagógica o práctica profesional docente; $h$ ) establecer el régimen de acceso, permanencia y promoción del personal docente y no docente; i) designar y remover personal; j) establecer el régimen de admisión, permanencia y promoción de los estudiantes y el régimen de equivalencias; $\mathrm{k}$ ) revalidar títulos extranjeros; 1) fijar el régimen de convivencia; $\mathrm{m}$ ) desarrollar y aplicar los conocimientos impartidos; n) mantener relaciones educativas, científicas y culturales con instituciones del país y el extranjero; ñ) reconocer asociaciones estudiantiles. Para poder cumplimenta cada una de estas funciones previstas en el artículo 29 de la LES, los IUFFAA deben contar con la autorización del Estado Mayor de su Fuerza y del Ministerio de Defensa.

13 De acuerdo con el artículo 77 de la Ley, los IUFFAA, al igual que otros institutos universitarios, "establecerán su sistema de gobierno conforme a sus propios regímenes institucionales, no siéndoles de aplicación las normas sobre autonomía y sobre gobierno de las instituciones universitarias nacionales que prevé la presente ley". 
las máximas autoridades del gobierno del IESE, INUN y IUA son oficiales superiores en actividad designados por la conducción de cada una de las Fuerzas, pudiendo completarse sus cuadros de gestión (nivel de secretarios) con oficiales en actividad (como en el caso del IESE), oficiales retirados (INUN) o por personal civil y militar (IUA). Los IUFFAA tampoco disponen de autarquía económica y financiera, dado que sus asignaciones presupuestarias son definidas por las propias Fuerzas.

Teniendo en cuenta, entonces, el modo particular en que los IUFFAA se incorporaron al ordenamiento de la LES contando con márgenes de autonomía mucho más restringidos que el de otras instituciones universitarias, efectuaremos a continuación una presentación de las etapas de la evaluación de estos institutos y enunciaremos las principales cuestiones destacadas en los informes de evaluación institucional externa realizados por las comisiones de pares evaluadores de la CONEAU entre los años 2000 y 2010. De este modo, nos será posible identificar recomendaciones clave formuladas al IESE, INUN y IUA, las cuales consideraremos a la luz del análisis de las perspectivas de cambio y continuidad de los IUFFAA en relación con definiciones relativas a la caracterización de sus formas de gobierno y gestión, organización institucional, diseño de ofertas educativas, perfiles de sus docentes, sociabilidad de sus estudiantes, articulación e integración al interior del sistema educativo castrense y del sistema público de educación superior. Entendemos, pues, que el modo en que estas orientaciones hayan sido desatendidas, asimiladas o procesadas por los actores institucionales (autoridades militares y civiles, profesores, cadetes y alumnos) incidirá, en consecuencia, en la ponderación de los márgenes de autonomía y heteronomía pasados, actuales y potenciales que dispongan los actores castrenses en el control de los IUFFAA ${ }^{14}$.

\section{LOS PROCESOS DE EVALUACIÓN INSTITUCIONAL EN LOS IUFFAA}

Al igual que en otras instituciones universitarias, la evaluación institucional en los IUFFAA se ha llevado a cabo conforme las definiciones postuladas por la CONEAU en el documento "Lineamientos para la evaluación institucional" del año 1997. Dicho documento establece las etapas y procedimientos que deben cumplimentarse en la evaluación institucional: $1^{\circ}$ ) Acuerdo compromiso entre la institución universitaria y la CONEAU; $2^{\circ}$ ) Proceso de auto-

14 Cabe aclarar que en esta oportunidad, por razones de extensión del artículo, no nos ocuparemos de analizar situacionalmente las perspectivas y experiencias de estos actores de los IUFFAA. 
-evaluación; $3^{\circ}$ ) Preparación de la evaluación externa; $4^{\circ}$ ) Desarrollo de las actividades del comité de pares evaluadores; $5^{\circ}$ ) Aprobación del informe por la CONEAU y su presentación a la institución; $6^{\circ}$ Comentarios del rector; $7^{\circ}$ Aprobación del informe final, publicación y difusión del mismo con los comentarios del rector. También ese documento delimita las variables básicas a analizar por la comisión de pares evaluadores: 1) Docencia; 2) Investigación, desarrollo y creación; 3) Extensión, producción de tecnología y transferencia; 4) Gestión y gobierno (sólo para universidades nacionales ${ }^{15}$ ); 5) Recursos humanos; Infraestructura y recursos materiales; 6) Servicios de biblioteca, de información e informáticos; 7) Integración de la institución universitaria.

En el IESE el proceso de autoevaluación comenzó en 1997 y en 1998 se firmó con la CONEAU un acuerdo general que definía el marco para su desarrollo. Los equipos técnicos de la CONEAU asistieron a la conducción del IESE para promover instancias de sensibilización en la población docente y autoridades. La autoevaluación concluyó a fines de 1999. El primer Informe de Evaluación Externa del IESE fue elaborado por los pares evaluadores el Médico y Doctor en Filosofía César Lorenzano, el Abogado Ricardo Biazzi, Doctor Andrés Fontana y el Ingeniero Carlos Lerch. Por la CONEAU participaron como miembros responsables Doctor José Luis Cantini y Doctor Juan Carlos Hidalgo y como miembro del equipo técnico César Peón. Este informe fue realizado en 2000 y publicado en 2002. A su vez, Informe Final de Autoevaluación Institucional del IESE fue presentado en 2008. El segundo Informe de Evaluación Externa del IESE fue elaborado por los pares evaluadores Licenciado Daniel Toribio, Julio Lima y el Ingeniero Jorge Bettaglio ${ }^{16}$. Por la CONEAU participaron como miembros responsables Juan Carlos Geneyro y Luis María Fernández y como miembro del equipo técnico Elizabeth Ciccorossi. Este informe fue realizado entre 2008/2009 y publicado en abril/ septiembre de 2010.

En 1997 el INUN organizó talleres con especialistas de la CONEAU y actividades de sensibilización con vistas a iniciar la autoevaluación. Desde 2002 se desarrolló el proceso de autoevaluación institucional que implicó una etapa de concientización y organización (año 2003), diseño y desarrollo de un modelo cuantitativo (2003-2004) y otro cualitativo (2004) y, por último, la elaboración del informe de autoevaluación (2004). A fines de 2004 se firmó el acuerdo con la CONEAU y se presentó formalmente el informe en 2006. Por su parte, el Informe de Evaluación Externa del INUN fue elaborado por

15 Los IUFFAA también fueron evaluados en este item.

16 En temas relativos al área de bibliotecas la evaluadora externa fue Marcela Fushimi. 
los pares evaluadores Ricardo Gutiérrez, Doctor en Relaciones Internacionales Ángel Tello y Licenciado en Ciencias Físicas Walter Edgardo Legnani ${ }^{17}$. Por la CONEAU participaron como miembros responsables José Francisco Martín y Pedro Krotsch y como miembro del equipo técnico Marisa Coler. El informe fue realizado en 2006 y publicado en abril de 2007.

El proceso de auto-evaluación comenzó en el IUA en 1997 y en 1998 se celebró el acta acuerdo con la CONEAU. El Informe de Evaluación Externa del IUA fue elaborado por la comisión de pares evaluadores integrada por el Magíster Miguel Guerrero, Ingeniero Roberto Juan Herbstein y el PhD. Roberto Martínez Nogueira. Por la CONEAU participaron como miembros responsables el Doctor René Nicoletti y el Doctor Héctor Sauret, asesor el profesor Daniel Prieto Castillo y miembro del equipo técnico la licenciada Mariana Alonso Bra. El informe fue realizado en 2001 y publicado en julio de 2002.

No todos los miembros de las comisiones de pares evaluadores que intervinieron en las evaluaciones institucionales externas contaban con formación académica y trayectorias profesionales reconocidas en los estudios sobre la defensa nacional y su instrumento militar. De los que sí poseían esos conocimientos específicos en esas dos temáticas y en relaciones internacionales eran Andrés Fontana y Ángel Tello. En algunos casos la expertise venía dada por la trayectoria en el campo de las ingenierías (Carlos Lerch, Jorge Bettaglio, Roberto Juan Herbstein) y la física (Walter Edgardo Legnani), que tienen -como se ha visto arriba- una presencia significativa en la oferta de los IUFFAA. Y en el resto de los perfiles de los evaluadores externos priman sus trayectorias académicas y experiencias en el gobierno y en la gestión universitaria. En este sentido, las evaluaciones externas de los IUFFAA ponen de manifiesto -al igual que en las evaluaciones de otras instituciones universitarias públicas y privadas- tensiones entre la pertenencia disciplinar y la institucional de los pares evaluadores (MARQUINA, 2009). En el caso específico de los IUFFAA, sus autoridades militares y oficiales instructores tienden a considerar que los pares evaluadores han puesto en foco de análisis los componentes en la organización universitaria y en la formación académica científica y profesional general antes que la dimensión institucional castrense y en los componentes teóricos y prácticos específicos de la formación militar.

A partir de la revisión de los informes de evaluación externa de las comisiones de la CONEAU, identificamos las siguientes recomendaciones de los pares evaluadores:

17 En temas relativos al área de bibliotecas la evaluadora externa fue Celia María Molina. 
a) Consolidar una planta docente calificada, con saberes actualizados, con antecedentes de docencia e investigación, mayor dedicación en la institución, postgrado e inserción en otras universidades nacionales y privadas, y que acceda a los cargos por régimen de concursos universitario. Crear también un régimen docente que no tenga la rigidez del actual sujeto a lo estipulado por el Estatuto del Personal Civil de las Fuerzas Armadas, contemplando una carrera normalizada que establezca la necesidad del perfeccionamiento. Producir la renovación competitiva de la planta docente y evitar los riesgos de su auto-reproducción.

b) Desarrollar un régimen de docentes-investigadores para constituir áreas de investigación. Las actividades de investigación y desarrollo deberían llevarse a cabo en ambientes institucionales adecuados, con una política que defina prioridades, bases y condiciones para el establecimiento de grupos y centros de investigación con al finalidad de formar recursos humanos de referencia para la institución, las Fuerzas y otras instituciones con intereses afines.

c) Crear un auténtico claustro de profesores, con elección de representantes por sus pares y con arreglo a criterios propios.

d) Desarrollar un sistema de gestión académica con funcionarios civiles especializados y de carrera, a fin de evitar que la alta rotación de los oficiales jefes y superiores en sus carreras profesionales impacte negativamente sobre los institutos de formación de oficiales toda vez que ellos son trasladados a otros destinos. Generar instancias de integración e intercambio de esos funcionarios civiles con los de las universidades nacionales.

e) Realizar una transición hacia modelos de gestión académica que contemple modelos deliberativos, participativos y horizontales en aquellas áreas donde sea posible concretarlos. El sistema interno de jerarquías que se homologa al de la organización de las Fuerzas Armadas no se corresponde plenamente con la naturaleza y demandas propias de la vida universitaria.

f) Incorporar los planes de estudios materias de contenido humanístico relacionándolas con las necesidades específicas de la formación profesional, facilitando a los cadetes mayor cantidad de tiempo destinado al estudio y que, en lo posible, que éstas materias puedan cursarse en el ámbito de universidades nacionales a fin de evitar 
restringir las instancias de formación exclusivamente a los límites internos de la academia militar.

g) Promover una valorización positiva equivalente entre la adquisición de saberes académicos generales y saberes y prácticas profesionales militares específicos, evitando cualquier forma de ponderación diferencial de estos últimos sobre los primeros.

h) Producir readecuaciones en el diseño de los tiempos dedicados a la formación de los cadetes a fin de compatibilizar adecuadamente los requerimientos demandados en la formación de un profesional universitario conjuntamente con un militar.

i) Definir programas de bienestar estudiantil y formación cívica que aseguren una mayor participación en relaciones horizontales de los cadetes en la vida universitaria de los institutos, promoviendo la formación de clubes o centros de estudiantes.

j) Generar mayores niveles de coordinación e integración entre los institutos de formación de oficiales de las tres Fuerzas Armadas, permitiendo así no sólo la optimización de recursos sino procurando el desarrollo del accionar militar conjunto.

k) Desarrollar redes interinstitucionales con universidades e instituciones científicas civiles con fines de intercambio de conocimientos, formación, investigación y de extensión/servicios/transferencia.

1) Consolidar el reconocimiento social y académico de los institutos de formación de oficiales, asentándolo en el prestigio de las capacidades científicas y tecnológicas de las Fuerzas Armadas -sus ventajas competitivas en el sistema de educación superior- y en su direccionamiento en función de las necesidades y demandas de la sociedad.

De la lectura de este sucinto registro de análisis y recomendaciones de los pares evaluadores en los informes de evaluación institucional externa de la CONEAU, puede apreciarse la tendencia planteada a los institutos militares de nivel universitarios en favor de la afirmación de formas de determinación heterónomas que interactúan (no necesariamente en forma solidaria) con la afirmación de su dependencia respecto de otras instancias jerárquicas superiores de cada Fuerza y del Ministerio de Defensa. Veamos más en detalle esta cuestión. 
Respecto de las actividades de docencia/investigación/extensión/transferencia los pares evaluadores enfatizaban la necesidad de incorporar a los IUFFAA a docentes e investigadores con trayectoria e inserción en otras universidades nacionales y privadas, a fin de evitar que la planta de profesores se complete mediante mecanismos de "auto-reproducción", una expresión de los evaluadores externos que puede ser entendida en términos de una cobertura que privilegiaba el reclutamiento de personal militar retirado y/o de profesores civiles procedentes sólo de universidades privadas y de algunas públicas con las cuales las academias castrenses mantienen lazos institucionales y personalizados de larga duración. También los evaluadores señalaban que es preciso crear, por un lado, una estructura de la organización docente que no estuviese organizada en torno del criterio escolar de asignación de horas-cátedra; y, por otro lado, generar un régimen competitivo de carrera docente que estimule el perfeccionamiento continuo y el desarrollo de la investigación.

En cuanto al gobierno y la gestión universitaria en los IUFFAA, las recomendaciones de los pares evaluadores rompen con la ponderación de modelos propios de la organización castrense, sugiriendo la constitución de un claustro docente, la adopción de un sistema de representación de pares y la implementación de un esquema de gestión universitaria a cargo de personal civil especializado y no de oficiales jefes o superiores carentes de conocimientos específicos en gestión educativa y sometidos a un régimen de traslados y cambios de destinos que discontinúan la labor institucional. En relación con la formación de recursos humanos en las instituciones de formación básica de oficiales, los pares evaluadores observaban que se requiere alcanzar un mayor equilibrio entre el esfuerzo destinado a la formación académica general y a la formación técnico-militar específica de los cadetes, evitando ponderar esta última en desmedro de la primera. Destacaban además que la aplicación de un modelo a sociabilidad universitaria demanda la delimitación de mayor tiempo de estudio extra-áulico, actividades áulicas menos escolarizadas, disponibilidad de tiempo libre, creación de un claustro estudiantil con organizaciones corporativas de representación e, incluso, la concurrencia de los cadetes a cursos en universidades nacionales. Asimismo, los pares evaluadores sostuvieron que era necesario profundizar la integración de los IUFFAA en el sistema universitario nacional y, más ampliamente, en el de ciencia, tecnología e innovación, generando proyectos con otras instituciones, participando en programas universitarios de docencia, investigación y servicios/transferencia/ extensión desde la particular contribución que estos institutos castrenses pueden aportar al sistema de educación superior y a la sociedad, cumpliendo las 
restricciones que sobre los mismos impone la Ley de Reestructuración de las Fuerzas Armadas.

Por último señalemos que desde la segunda mitad de la década de 1990 hasta el presente los IUFFAA han acreditado las siguientes carreras de grado de "interés público" que gravitan, fundamentalmente, en el campo de las ingenierías y pueden ser cursadas por civiles y militares. El IESE concentra la oferta de estas carreras en la Escuela Superior Técnica, habiendo concretado su acreditación en 2003, su primera re-acreditación en 2007 y actualmente está en curso una nueva instancia de acreditación: Ingeniería Mecánica orientación Mantenimiento (Resolución 587/03 y 725/07), Ingeniería Mecánica orientación Automotores (Res.586/03 y 729/07), Ingeniería Mecánica orientación Armamentos (Res.585/03 y 724/07), Ingeniería Electrónica (Res.584/03 y 726/07), Ingeniería Química (Res.588/03 y 728/07) e Ingeniería Civil (Res.583/03 y 727/07). El IUA, en tanto, inscribe sus carreras de "interés público" en la Facultad de Ingeniería: Ingeniería Electrónica (Res.320/05 y 726/09), Ingeniería Mecánica Aeronáutica (Res.734/05), Ingeniería en Telecomunicaciones (Res.662/08) e Ingeniería en Mecánica Aeronáutica (Res.725/09). El INUN no posee este tipo de carreras; quizá deba explorarse a modo de hipótesis si la estrecha relación institucional y personalizada que mantienen históricamente oficiales de la Armada Argentina con el Instituto Tecnológico de Buenos Aires (ITBA), un instituto universitario privado creado en 1961, constituye un motivo plausible por el cual esta Fuerza no ha desarrollado en su sistema educativo una oferta de carreras de grado ligada a las ingenierías ${ }^{18}$.

Las ofertas de posgrado de los IUFFAA también se han sometido igualmente a los proceso de acreditación de la CONEAU. En el caso del IESE ha acreditado las Maestrías en Estrategia y Geopolítica (Res.786/99), en Historia de la Guerra (Res.787/99 y 476/07), en Defensa Nacional (Res.227/00 y $615 / 10)^{19}$, en Seguridad e Higiene (Res.234/00), en Transporte (Res.233/00), en Gestión Tecnológica (Res.031/04), Geopolítica (Res.441/10); y las Especializaciones en Derecho Militar (258/99), en Gestión Ambiental (Res.365/03) y Criptografía y Seguridad (Res.241/04). Todas se ofrecen en las unidades académicas de la Escuela Superior de Guerra y la Escuela Superior Técnica. Por su parte, el INUN en su sede académica de la Escuela Superior de Guerra

18 El ITBA ofrece las siguientes ingenierías: Electrónica, Eléctrica, Mecánica, Química, Informática, Industrial, Naval y en Petróleo, además de la Licenciatura en Administración y Sistemas.

19 La Maestría en Defensa Nacional la certifica el IESE pero se brinda en la escuela de defensa nacional que depende de la Subsecretaría de Formación del Ministerio de defensa (es decir, no es una unidad académica del IESE y tampoco depende orgánicamente de la Dirección General de Educación del Ejército). 
Naval las Maestrías en Estudios Estratégicos (Res.848/99 y 881/09), en Gestión Logística (Res.399/03) y en Dirección de las Organizaciones (Res.319/04). Por último el IUA cuenta en la unidad académica Facultad de Ingeniería una Maestría en Ciencias de la Ingeniería (Res.981/05) y las Especializaciones en Análisis de la Inteligencia Estratégica ${ }^{20}$, Sistemas Embebidos ${ }^{21}$ y en Seguridad Informática ${ }^{22}$.

\section{REFLEXIONES FINALES}

La transformación de las academias militares en institutos universitarios y su incorporación al sistema de educación superior estimuló desde la década de 1990 el desarrollo de experiencias "civilianizadoras"-según la expresión de Morris Janowitz (1974)- en la educación castrense; o, en otras palabras, la incorporación de lógicas y prácticas educativas tomadas del mundo civil que heteronomizaron el ámbito militar. Esos cambios redundaron en una mayor apertura de las Fuerzas Armadas para que civiles estudien en sus institutos y para que militares cumplimenten estudios de grado y posgrado que no forman parte del plan de carrera de los oficiales, pero que bien pueden redundar en beneficios para las Fuerzas y para las personas que los cursan.

En 1997 los IUFFAA se plegaron a las orientaciones de las políticas de evaluación definidas a partir de la sanción de la LES y sometieron sus posgrados y carreras de grado de "interés público" a periódicas acreditaciones por parte de la CONEAU. Dicha decisión fue impulsada activamente por la conducción de las Fuerzas Armadas no sólo como un modo de adecuarse a la normativa vigente, sino buscando obtener mayor reconocimiento y legitimidad social. Más recientemente, desde 2007 la conducción civil de la defensa en el área educativa -la Subsecretaría de Formación- ha alentado la profundización de esas transformaciones institucionales y curriculares de los IUFFAA. Particularmente el IESE ha concretado dos evaluaciones externas en 2000-2002 y 2008-2010. Y debe decirse al respecto que la reiteración de las recomendaciones arriba señaladas por parte de la comisión de pares evaluadores en ambos informes (coincidentes además con aquellas indicadas en los informes externos del INUN y al IUA), permite suponer que existe un núcleo

20 Esta especialización se cursa en el Instituto de Inteligencia de las Fuerzas Armadas con sede en la ciudad de Buenos Aires.

21 Esta Especialización en Sistemas Embebidos fue presentada ante el Ministerio de Educación, Ciencia y Tecnología (Exp. 4956/07) y el proyecto de carrera fue aprobado por la CONEAU.

22 En el marco de lo establecido por la Ord. N ${ }^{\circ} 56$ de la CONEAU) se aprobó el dictamen correspondiente al proyecto de carrera 10.322/09: Especialización en Seguridad Informática. 
duro de la cultura institucional de los institutos militares que hasta el momento permanece inmodificado a pesar de su participación en sucesivos procesos de autoevaluación y de evaluación institucional externa. Tal situación -que debería investigarse enfocando el estudio de perspectivas y experiencias de los actores sociales de los IUFFAA- confirmaría la existencia de amplios márgenes de autonomía institucional.

Ahora bien, la persistencia de esa autonomía en modo alguno es un fenómeno sólo expresivo de rasgos exclusivos o privativos de los actores del sistema educativo castrense, ni un inequívoco indicador de la persistencia de un esencial atavismo antidemocrático militar. Sonia Araujo (2007) ha observado en este sentido que las actitudes conservadoras y de resistencia al cambio se presentan en distintos procesos de evaluación universitaria, asumiendo formas diferentes de acuerdo a los perfiles corporativos y disciplinares de los actores sociales implicados, a la distribución del poder institucional y las formas de organización adoptadas en cada caso. En otras palabras, los procesos de evaluación institucional y de acreditación de carreras en universidades nacionales y privadas, corroboran que los actores universitarios activan diversas formas y grados de resistencia, de resignificación y/o de apropiación de las orientaciones que informan dichos procesos ${ }^{23}$. En consecuencia deberían determinarse cuáles son las características específicas que asume esa autonomía en los IUFFAA y cuáles las que comparte con instituciones universitarias civiles. Por último, resulta necesario pensar también en qué medida esa autonomía continúan reproduciéndose cuando las autoridades civiles no orientan decididamente las políticas de educación militar o bien cuando lo hacen en forma intermitente o deficiente.

23 Pedro Krotsch, Antonio Camou y Marcelo Prati (2007) destacan que es necesario investigar las estrategias formales e informales de adopción, adaptación y/o resistencia desplegadas por los actores universitarios frente a las políticas de evaluación universitarias, evitando asumir su implementación como un proceso unilineal y no conflictivo desplegado por la Secretaría de Políticas Universitarias o la CONEAU sobre las universidades. Asimismo, también destacaron la necesidad de indagar empíricamente a nivel institucional la singularidad del impacto de esas políticas en distintas funciones universitarias (docencia, investigación, transferencia/extensión), en los niveles organizativos del sistema (por universidad, unidad académica, departamento, carrera), por disciplinas y en diferentes actores (autoridades, profesores, investigadores, graduados, estudiantes). 


\section{REFERENCIAS}

ALTBACH, Philip; McGILL PETERSEN, Patti. Educación superior en el siglo XXI. Desafío global y respuesta nacional. Buenos Aires: Biblos, 2000.

ARAUJO, Sonia. Evaluación institucional y cambio universitario. Un difícil proceso de reconstrucción. En: KROTSCH, P.; CAMOU, A.; PRATI, M. (Coords.). Evaluando la evaluación. Políticas universitarias, instituciones y actores en Argentina y América Latina. Buenos Aires: Prometeo, 2007. p. 69-94.

BADARÓ, Máximo. Militares o ciudadanos, la formación de los oficiales del Ejército Argentino. Buenos Aires: Prometeo, 2009.

CAMOU, Antonio. Los 'juegos' de la evaluación universitaria en la Argentina. Notas sobre las interacciones conflictivas entre Estado y universidad. En KROTSCH, P.; CAMOU, A.; PRATI, M. (Coords.). Evaluando la evaluación. Políticas universitarias, instituciones y actores en Argentina y América Latina. Buenos Aires: Prometeo Libros, 2007. p. 29-68.

CANELO, Paula. Consideraciones sobre la subordinación de las Fuerzas Armadas Argentinas durante los años noventa. En: PUCCIARELLI, A. (Coord.). Los años de Menem. La construcción del orden neoliberal. Buenos Aires: Siglo Veintiuno Editores, 2011. p.143-178.

CHIROLEU, Adriana. Los contextos que enmarcan la reforma de la ley de educación superior: entre desafíos y oportunidades. En: MARQUINA, M.; SOPRANO, G. (Comps.). Ideas sobre la cuestión universitaria. Los Polvorines: Universidad Nacional de General Sarmiento, 2007. p. 37-56.

FREDERIC, Sabina; SOPRANO, Germán et al. La formación militar como formación moral: transmisión y adquisición de saberes teóricos y prácticos en las Fuerzas Armadas. En: FREDERIC, S.; GRACIANO, O.; SOPRANO, G. (Eds.). El Estado argentino y las profesiones liberales, académicas y armadas. Rosario: Prohistoria, 2010. p. 387-420.

FREDERIC, Sabina ; SOPRANO, Germán. Políticas de educación superior y transformaciones de los institutos militares en la Argentina (de 1990 al presente). En: ALVES, V. C.; SOUZA MOREIRA, W. de; ARIAS NETO, J. 
M. (Org.). A defesa e a segurança na América do Sul. São Paulo: Mercado das Letras, 2011. p. 215-234.

JANOWITZ, Morris. The professional soldier. A social and political portrait. London: The Free Press / Collier Macmillan, 1974.

KROTSCH, Pedro. Educación superior y reformas comparadas. Bernal: Universidad Nacional de Quilmes, 2001.

KROTSCH, Pedro; PUIGGROS, Adriana. Universidad y evaluación. Estado del debate. Buenos Aires: Aique, 1994.

KROTSCH, Pedro; CAMOU, Antonio; PRATI, Marcelo. Presentación. En: KROTSCH, P.; CAMOU, A.; PRATI, M. (Coords.). Evaluando la evaluación. Políticas universitarias, instituciones y actores en Argentina y América Latina. Buenos Aires: Prometeo, 2007. p. 13-27.

KROTSCH, Pedro; ATAIRO, Daniel; VARELA, Sebastián. De la simultaneidad e inrteracción en los procesos de construcción de la evaluación institucional: el caso de la UNICEN. En: KROTSCH, P.; CAMOU, A.; PRATI, M. (Coords.). Evaluando la evaluación. Políticas universitarias, instituciones y actores en Argentina y América Latina. Buenos Aires: Prometeo, 2007. p. 97-124.

LÓPEZ, Ernesto. Sudamérica: nueva inestabilidad y desajuste en la subordinación militar. Revista de la Sociedad Argentina de Análisis Político, Buenos Aires, v. 2, n. 1, p.165-183. 2004.

MARQUINA, Mónica. Académicos como evaluadores de instituciones y carreras universitarias en Argentina: la experiencia de una década. En: Marquina, M.; MAZZOLA, C.; SOPRANO, G. (Comps.). Políticas, instituciones y protagonistas de la universidad argentina. Buenos Aires: Universidad Nacional de General Sarmiento / Universidad Nacional de San Luis / Prometeo, 2009. p. 75-94.

MAZZOLA, Carlos. Crisis institucional en la universidad argentina. Avaliação, Campinas; Serocaba, v.13, n.1, p. 89-100. 2008.

MONTENEGRO, Esteban Germán. El marco normativo y doctrinario de la defensa nacional. Revista de la Defensa Nacional, n. 1, p. 14-27, 2007. 
NEAVE, Guy. Educación superior: historia y política. Estudios comparativos sobre la universidad contemporánea. Barcelona: Gedisa, 2001.

O'DONNELL, Guillermo; SCHMITTER, Philippe; WHITEHEAD, Laurence. Transiciones desde un gobierno autoritario. Barcelona: Paidós, 1994.

PEÓN, César; PUGLIESE, Juan Carlos. Análisis de los antecedentes, criterios y procedimientos para la evaluación institucional universitaria en la Argentina (1996-2002). En: BARSKY, O.; SIGAL V.; DÁVILA, Y.M. (Coords.). Los desafíos de la universidad argentina. Buenos Aires: Universidad de Belgrano; Siglo Veintiuno, 2004. p. 489-520.

PÉREZ RASETTI, Carlos. Pertinencia y condición universitaria en los Institutos Universitarios de las Fuerzas Armadas. Revista Manifiesto, San Miguel de Tucumán, n. 1, 2008.

PION-BERLIN, David. Militares y democracia en el nuevo siglo. Cuatro descubrimientos inesperados y una conclusión sorprendente. Nueva Sociedad, Buenos Aires, n. 213, p. 50-63. 2008.

SAIN, Marcelo Fabián. Los votos y las botas. Estudios sobre la defensa nacional y las relaciones civil-militares en la democracia argentina. Buenos Aires: Prometeo, 2010. 
